\title{
Attentional Selection and Action Selection in the Ventral and Orbital Prefrontal Cortex
}

\author{
Matthew F. S. Rushworth, Mark J. Buckley, Patricia M. Gough, Iona H. Alexander, Diana Kyriazis, \\ Kathryn R. McDonald, and Richard E. Passingham \\ Department of Experimental Psychology, University of Oxford, Oxford OX1 3UD, United Kingdom
}

Different accounts of the ventral and orbital prefrontal cortex $(\mathrm{PFv}+\mathrm{o})$ have emphasized either its role in learning conditional rules for action selection or the attentional selection of behaviorally relevant stimuli. Although the accounts are not mutually exclusive, it is possible that the involvement of $\mathrm{PFv}+\mathrm{o}$ in conditional action selection is a consequence of its role in selecting relevant stimuli or that its involvement in attentional selection is a consequence of the conditional rules present in many attentional paradigms. Five macaques learned a conditional action-selection task in which the difficulty of identifying the stimulus relevant for guiding action selection was varied in a simple manner by either altering its distance from the action or presenting additional distracting stimuli. Simply increasing the spatial separation between the instructing stimulus led to slower responses. Experiment 1 showed that bilateral PFv $+o$ lesions impaired conditional action selection even when attentional demands were kept to a minimum, but there was evidence that the impairment was exacerbated by manipulating stimulus selection difficulty. Experiment 2 confirmed the importance of PFv $+o$ for conditional action selection even when stimulus selection difficulty was minimal. Experiments 3 and 4 demonstrated that the action-selection impairment was significantly increased by making identification of the behaviorally relevant stimulus difficult. $\mathrm{PFv}+\mathrm{o}$ is central to the use of conditional rules when selecting courses of action, but conditional rules are also represented in premotor and striatal regions. A special contribution of $\mathrm{PFv}+\mathrm{o}$ may be initial selection of behaviorally relevant stimuli.

Key words: prefrontal cortex; conditional motor learning; attention; macaque; lesion; action selection

\section{Introduction}

One account of ventral and orbital prefrontal cortex $(\mathrm{PFv}+\mathrm{o})$ has emphasized its involvement in the learning of arbitrary conditional rules for linking stimuli and actions (Rushworth and Owen, 1998; Passingham et al., 2000; Wise and Murray, 2000). In many situations, the selection of the appropriate course of action is conditional on the presence of one or other instruction, stimulus, or context. It is thought that $\mathrm{PFv}+\mathrm{o}$ is central to the learning of conditional associations; $\mathrm{PFv}+\mathrm{o}$ lesions impair the learning of conditional associations while leaving other types of learning, such as discrimination learning, relatively unaffected (Parker and Gaffan, 1998; Bussey et al., 2001). Neural activity in PFv+o is modulated when conditional rules guide choices (White and Wise, 1999; Wallis et al., 2001). The particular contribution made by $\mathrm{PFv}+\mathrm{o}$ remains unclear; conditional learning also depends on a network of striatal, premotor, and periarcuate regions (Petrides, 1985; Wallis and Miller, 2003; Brasted and Wise, 2004; Nixon et al., 2004; Pasupathy and Miller, 2005). The first aim of the current experiments was to test the hypothesis that $\mathrm{PFv}+\mathrm{o}$ is

Received July 5, 2005; revised 0ct. 25, 2005; accepted 0ct. 26, 2005.

This work was supported by the Wellcome Trust and Royal Society (M.F.S.R., M.J.B.). We are very grateful to D. Gaffan for advice and encouragement.

Correspondence should be addressed to Matthew Rushworth, Department of Experimental Psychology, South Parks Road, 0xford 0X1 3UD, UK. E-mail: matthew.rushworth@psy.ox.ac.uk.

D0I:10.1523/JNEUROSCI.2765-05.2005

Copyright $\odot 2005$ Society for Neuroscience $\quad$ 0270-6474/05/2511628-09\$15.00/0 particularly important for attentional selection of the stimulus information that is relevant for guiding conditional choices.

A distinct account of $\mathrm{PFv}+\mathrm{o}$ has emphasized its role in attentional selection of behaviorally relevant stimuli (Sakagami and Niki, 1994; Rainer et al., 1998; Everling et al., 2002; Lebedev et al., 2004) or behaviorally relevant dimensions of stimuli (Dias et al., 1996; Brass and von Cramon, 2004). It is possible that only one of the two descriptions of $\mathrm{PFv}+\mathrm{o}$ function, in terms of either conditional action selection or attentional selection, is correct. The $\mathrm{PFv}+\mathrm{o}$ involvement in conditional action selection could be a consequence of its primary role in selecting behaviorally relevant stimuli. Alternatively, it might be argued that $\mathrm{PFv}+\mathrm{o}$ involvement in many attention paradigms is a consequence of the arbitrary rules that govern performance in such tasks. The second aim of the current experiment was therefore to examine the evidence for the two accounts of PFv+o.

The importance of $\mathrm{PFv}+\mathrm{o}$ for visuospatial conditional associations was assessed while the difficulty of selecting the behaviorally relevant stimulus information was varied. Stimulus selection can be made more difficult by spatially separating the relevant stimulus information from the response position (Schrier and Harlow, 1957); it is difficult for monkeys to appreciate the behavioral relevance of a stimulus when the action is made at a distance. Five macaques were taught to respond to response boxes on the left or right of a touch-screen monitor (see Fig. 1a). The correct action was conditional on the presence of a pair of identical visual stimuli. The behavioral relevance of the 

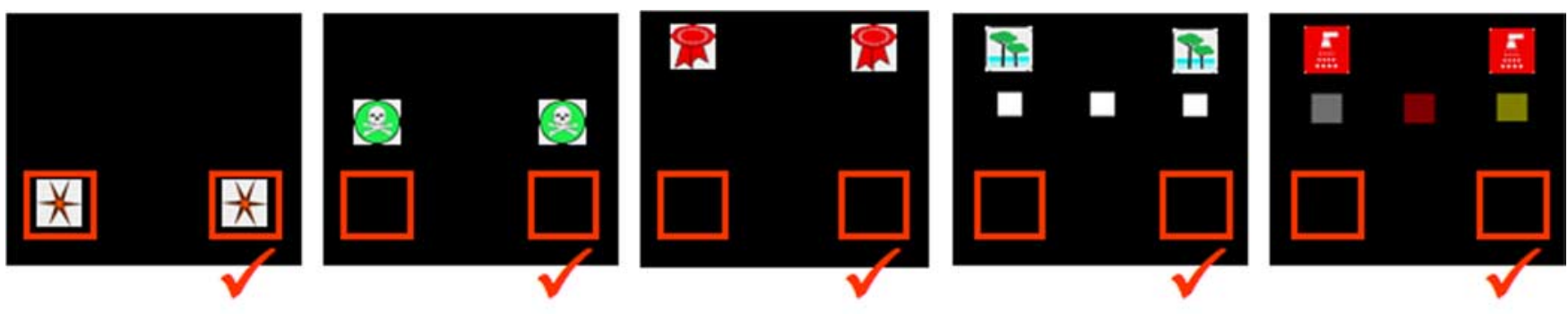

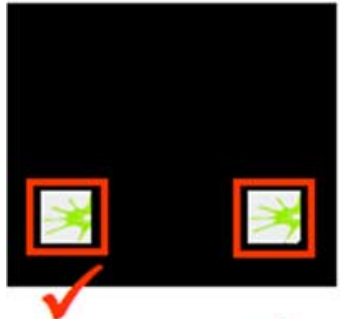

a)

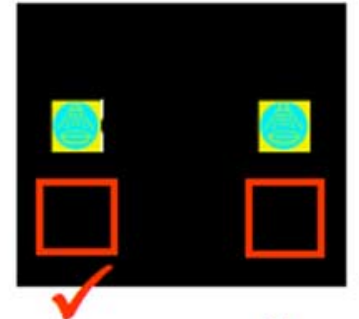

b)

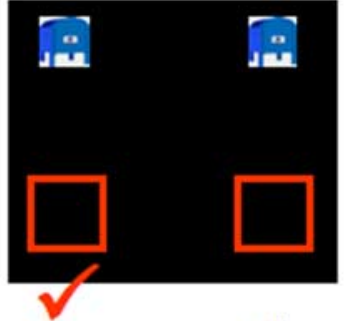

c)

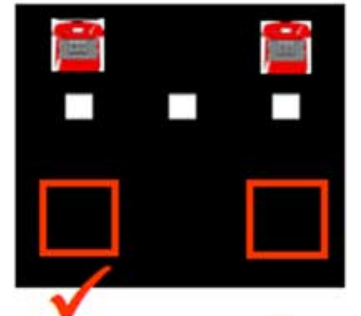

d)

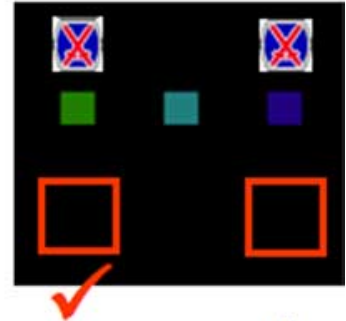

e)

Figure 1. Two examples of the touch-screen layout for trials of each condition: $\boldsymbol{a}$, inside; $\boldsymbol{b}$, intermediate; $\boldsymbol{c}$, far; $\boldsymbol{d}$, white distractor; and $\boldsymbol{e}$, color distractor. In all cases, the monkeys made responses to either the left or the right response boxes, which were indicated by flashing red squares. Two copies of the same visual stimulus were shown on the screen on every trial. The visual stimuli instructed responses to either the box on the left or the right. The correct response is to the right in each of the example problems shown at the top of the figure, whereas the correct response is to the left for each example problem shown at the bottom. Instructing visual stimuli were present on every trial. On inside trials (a), the instructing visual stimuli were placed inside the response box, but they were moved farther away in the intermediate $(\boldsymbol{b})$ and far $(\boldsymbol{c})$ trials. On other trials, white distractors ( $\boldsymbol{d})$ or color distractors $(\boldsymbol{e})$ were also shown on the screen between the instructing stimuli and the response boxes.

stimuli was made readily apparent by placing them directly inside the response boxes on some trials. The behavioral relevance of the stimuli was less obvious on other trials when the stimuli were placed at a distance from the response boxes (see Fig. 1b,c) and when interposing distractors appeared between the stimuli and the response boxes (see Fig. 1d,e). The effect of PFv+o lesions on retention and learning of visuospatial conditional associations was then tested.

\section{Materials and Methods}

Subjects. Five male rhesus macaques between the ages of 3 and 6 years were used. The studies were conducted under project and personal licenses issued by the British Home Office and in accordance with the British Animal Scientific Procedures Act (1986) and the Guide for the Care and Use of Laboratory Animals (National Research Council).

$\mathrm{PFv}+\mathrm{o}$ lesions were made in two animals, and their preoperative and postoperative performance was compared with that of the remaining controls. By careful experimental design, we hoped to be able to show statistically significant differences in performance, as others have done, by just analyzing the performance of two animals with lesions (Schiller and Chou, 1998; De Weerd et al., 1999, 2003; Buffalo et al., 2005). A subsequent experiment (experiment 4) further examined the nature of the $\mathrm{PFv}+\mathrm{o}$ deficit by comparing the preoperative and postoperative performance of the remaining three animals.

Procedure. The animals were taught tasks involving conditional associations between stimuli and responses. There are a large variety of such tasks in which the responses may be either a manual or oculomotor action, but critical features include the stipulation that the association between the stimulus and responses is a learned and arbitrary one and that the response that is correct is not constant but varies from trial to trial because it is conditional on some other factor (Passingham, 1993; Chen and Wise, 1995; Murray et al., 2002). Thus, delayed matching and nonmatching tasks are examples of conditional tasks, because monkeys and people are able to learn to make both matching and nonmatching responses, although different species may have different predispositions toward the use of matching or nonmatching rules (Passingham, 1993; Wallis et al., 2001; Murray et al., 2002; Bunge et al., 2003). In the present experiment, animals were taught visuospatial conditional tasks.

On each trial, monkeys chose between actions to one of two response boxes with distinct spatial positions. The correct spatial choice was conditional on the identity of visual stimuli presented on the screen. On some trials ("inside" condition), the stimuli for guiding action selection were presented at the positions to which the actions would be directed (Fig. 1a). This meant that there was no spatial separation between the instructing stimulus and the action. Because actions could be made to either one of two positions, however, it was necessary to present a pair of stimuli so that one stimulus could be placed at each possible action location.

On other trials, the instructing stimuli were spatially separated from the response boxes and placed at an "intermediate" (Fig. 1b) or "far" (Fig. 1c) position. On some trials, three "white distractors" (Fig. 1d) or three different "color distractors" (Fig. 1e) were presented between the relevant stimuli and the response boxes.

Each correct action was reinforced by a $190 \mathrm{mg}$ food pellet. In experiments $1-3$, the pellets were delivered in one of two food wells placed on either side of the touch screen close to the response boxes. On correct trials, the food pellet was delivered to the well next to the response box that had been touched. This manipulation ensured the smallest possible spatial separation between the target of the action and the contingent reward, although the separation between the stimulus and the action positions was varied from trial to trial.

After making a correct response, the instructing stimulus on the same side of the screen as the correct response box and the correct response box remained on the screen for an additional $2000 \mathrm{~ms}$. When an incorrect action was made, no reward was given and both instructing stimuli were immediately removed from the screen. A trial rerun correction procedure was used during training but not at any stage during the acquisition of both preoperative and postoperative data in experiments $1-3$. An intertrial interval of $8500 \mathrm{~ms}$ separated the end of one trial from the beginning of the next. The intertrial interval could neither begin nor end if the animal were touching the touch screen. On each day of testing in experiments $1-3$, each animal's session continued until 100 trials had been performed correctly.

It should be noted that all the errors reported were the result of the monkey touching the incorrect stimulus, and none were simply the consequence of the monkey making an inaccurate movement, in the sense of failing to touch either target.

Apparatus. All stimuli were presented on a visual display unit $(\sim 39 \mathrm{~cm}$ 
across and $28 \mathrm{~cm}$ high) fitted with a touchsensitive screen and connected to a personal computer. During testing, animals were brought in wheeled cage to a position $20 \mathrm{~cm}$ away from the monitor so that it could be touched easily.

Two red outline squares $(7.5 \mathrm{~cm}$ across) remained present on the lower left and right of the screen $(0.5 \mathrm{~cm}$ from the bottom of the screen and $4 \mathrm{~cm}$ from the left-hand and right-hand edges of the screen) throughout the intertrial interval. On each trial, the red-outlined squares flashed on and off at a rate of $5 \mathrm{~Hz}$, and a pair of identical stimuli appeared on the screen. The square stimuli were $6 \mathrm{~cm}$ across, and they were separated by $17.5 \mathrm{~cm}$. The stimuli could therefore fit inside the response boxes in the inside condition. The size of stimuli and their separation from one another remained constant in the intermediate, far, white distractor, and color distractor conditions. The food wells were $11.5 \times 8 \mathrm{~cm}$ and, at their nearest point, were 3 $\mathrm{cm}$ from the response box.

Initial training. All five animals were initially taught a pair of visuospatial conditional problems in the inside condition (Fig. 1a). Following the definition used by Bussey et al. $(2001,2002)$ "problem" refers to a single stimulus-action association. To solve a particular problem, the monkey would have to learn whether to direct actions to the left or right whenever the stimulus was shown. Each problem was always presented in the context of a "pair" or more of problems, and no problem was ever tested in isolation A "problem pair" always consisted of one problem for which the correct action was made to the left response box and one problem for which the correct action was made to the right response box. After animals reached a criterion of $90 \%$ correct responding, they were taught three more problem pairs to the same $90 \%$ correct criterion.

On the fifth problem pair, testing conditions were changed. On the first trial, the stimuli were presented $\sim 6 \mathrm{~cm}$ higher so that only the very bottom of the stimuli partly overlapped the response boxes as in the standard inside condition. After each correct response, the stimuli were shifted $\sim 0.5 \mathrm{~mm}$ upwards on the screen so that, by the end of the session, the conditions approximated those used in the intermediate condition (Fig. 1b). The animals were taught subsequent intermediate and far problems with the bottom edge of stimuli separated from the top edge of the response boxes by 4.8 and $12.2 \mathrm{~cm}$ from the outset of testing. The white distractor and color distractor conditions were then initially introduced by presenting distractors on a previously learned far condition problem pair. Subsequent white and color distractor problems were learned with distractors present from the start. The distractors were $4 \mathrm{~cm}$ squares, and their lower edge was $6 \mathrm{~cm}$ above the upper edge of the response boxes. The two lateral distractors were positioned so that their centers were at the same horizontal position as the horizontal centers of the instructing stimuli and the response boxes.

Animals were then presented with sessions with increasing number of problems up to a maximum of 10 problems (five problem pairs), and testing continued until a criterion of $>85 \%$ average correct performance was reached over 200 rewards (i.e., over $2 \mathrm{~d}$ ). A group of 10 problems is subsequently referred to as a "10-problem set." Each 10-problem set contained an example of an inside, an intermediate, a far, a white distractor, and a color distractor problem pair.

Surgery and histology. All surgery was performed under sterile conditions with sodium pentobarbitone anesthesia and with the aid of a binocular operating microscope. Tissue was retracted in anatomical layers, and a bilateral bone flap was removed. The lesions were made by aspira- tion with a fine-gauge sucker. The wound was closed in anatomical layers. At least 3 weeks were allowed for recovery before testing resumed.

In experiments $1-3$ and in experiment 4 , the intention was to make $\mathrm{PFv}+\mathrm{o}$ lesions similar to those that have been shown previously to cause a reliable impairment on the conditional action-selection tasks (Parker and Gaffan, 1998; Bussey et al., 2001). Bussey et al. (2001) attempted to remove most of the tissue that received inputs from visual sensory areas in the temporal lobe. They referred to the lesion as a $\mathrm{PFv}+\mathrm{o}$ lesion because the lesion included the inferior convexity of the ventral prefrontal cortex and extended to include the lateral orbital cortex lateral to the medial orbital sulcus. The boundaries of the intended lesion were as follows (Fig. 2a,b). The dorsal boundary was $5 \mathrm{~mm}$ ventral to the principal sulcus. Dorsally, the caudal boundary of the lesion extended along an imaginary line from the caudal end of the principal sulcus to the most rostral point of the inferior limb of the arcuate sulcus. From this point, the lesion extended onto the orbital surface, in which the caudal boundary extended to the caudal end of the lateral orbital sulcus and then the caudal end of the medial orbital sulcus. The ventromedial boundary was intended to be lateral to the medial bank of the medial orbital sulcus. The rostral boundary was the set of lines joining the most rostral points of the medial and lateral orbital sulci and the principal sulcus.

When the animals had completed their testing, they were anesthetized with sodium pentobarbitone and perfused with $90 \%$ saline and $10 \%$ formalin. The brains were then removed and placed in 10\% sucrose formalin until they sank. The brains were then blocked in the coronal plane at the level of the most medial part of the central sulcus. Each brain was then cut in $50 \mu \mathrm{m}$ coronal sections. Every 10th section was retained for analysis and stained with cresyl violet. Coronal sections through the frontal lobes, including the lesions, of the animals used in experiments 


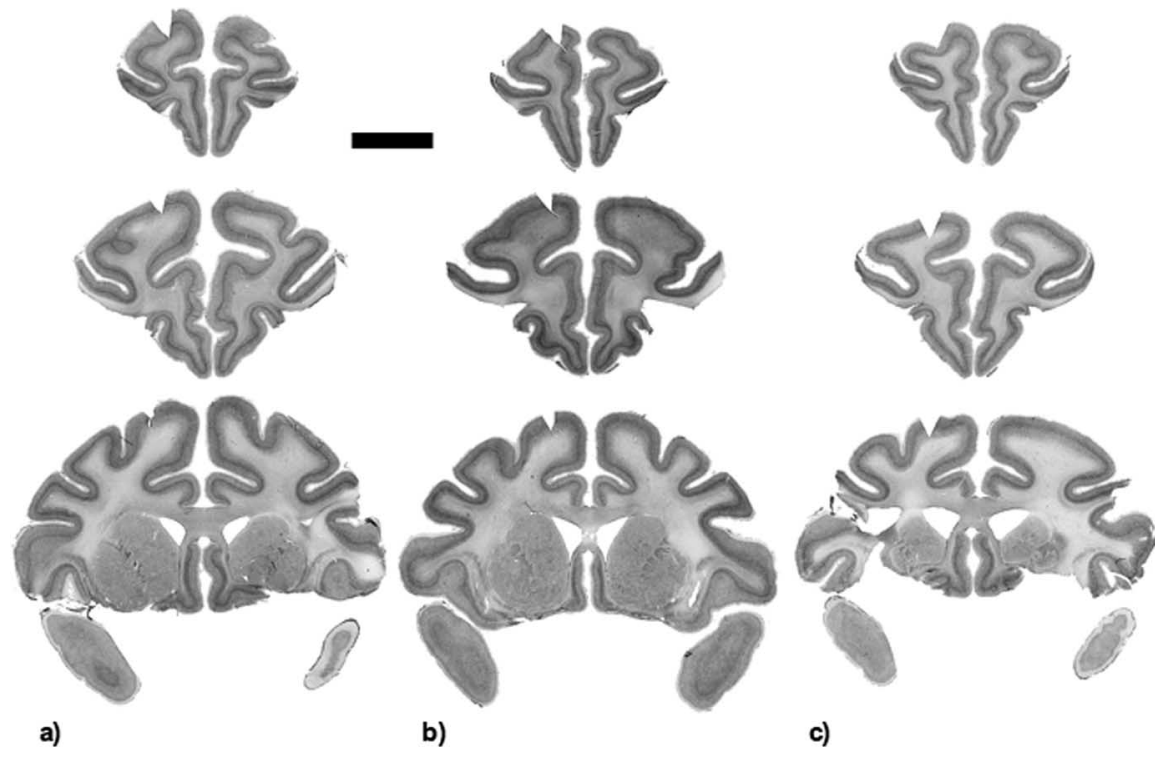

Figure 3. Three coronal sections taken through the lesions in animals PFV3 $(\boldsymbol{a}), \operatorname{PFV} 4(\boldsymbol{b})$, and PFV5 (c). The sections were taken at approximately the same levels as in Figure 2 . Scale bar: $\boldsymbol{a}, \boldsymbol{b}, \boldsymbol{c}, \sim 10 \mathrm{~mm}$ distance.

1-3 are shown in Figure 2, $c$ and $d$. The histology for the animals used in experiment 4 is shown in Figure 3. As can be seen, for the most part the lesions were made as intended.

Analyses. Results were analyzed with two- or three-way ANOVAs using a between-subject factor of group $(\mathrm{PFv}+\mathrm{o}$ lesion or control) and within-subject factors of period (preoperative testing period or postoperative testing period), distance (inside, intermediate, and far conditions), or distractor presence (far, white distractor, and color distractor conditions) and problem (for example, first, second, or third 10-problem set in experiment 1). Parametric ANOVAs were used because there are widely accepted parametric procedures for performing mixed-model multifactorial analyses, and there are no generally accepted nonparametric alternatives. Although parametric ANOVA procedures are robust even when there are differences in variance, the impact of such differences when they are large (experiments 1 and 4) was reduced by log transformation of data before analysis. In addition, the Huynh-Feldt correction procedure for violation of assumptions concerning variance was used when testing for the significance of repeated-measure factors.

Experiment 1: preoperative and postoperative comparison of $\mathrm{PFv}+\mathrm{ole}$ sion on stimulus selection and conditional action selection. All five animals were tested on the retention of three 10-problem sets on two occasions 4 weeks apart. As explained above, each 10-problem set consisted of an inside, an intermediate, a far, a white distractor, and a color distractor problem pair. Each retention test comprised six sequential sessions, two for each 10-problem set. In other words, all five animals were tested on 30 problems composed of equal numbers of inside, intermediate, far, white distractor, and color distractor problems on two occasions. Bilateral $\mathrm{PFv}+\mathrm{o}$ lesions were made in two animals, PFV1 and PFV2, between the two testing periods. The three control animals received no testing during a period equivalent to the operated animals' recovery period.

Experiment 2a: the effect of PFv +o lesions on learning new conditional action-selection problems in the absence of any separation between stimuli and response boxes. The results of experiment 1 suggested that two conclusions might be drawn. First, PFv +o lesions impaired stimulus conditional action selection even when the instructing stimuli were not spatially separated from the response boxes. Second, $\mathrm{PFv}+\mathrm{o}$ lesions impaired performance to an even greater degree when the stimulus and action positions were separated. Taken in isolation, however, it was possible to dispute each of the two conclusions. The first and second conclusions were therefore further investigated in this experiment, $2 \mathrm{a}$, and in the next experiment, $2 \mathrm{~b}$, respectively.

Against the first conclusion, it might be contended that $\mathrm{PFv}+\mathrm{o}$ lesion performance was most impaired in the conditions that stressed selection of the relevant stimulus (far, white distractor, and color distractor). Because of the interleaved presentation of all the different trial types, a failure set might have generalized to even the easier inside and intermediate trials in the animals with $\mathrm{PFv}+\mathrm{o}$ lesions.

To test this possibility, all five animals were taught four new inside condition problem pairs. Each problem pair was taught separately and in the absence of any intermediate, far, white distractor, or color distractor trials to a criterion of $>85 \%$ average correct performance while obtaining 200 rewards (i.e., over 2 d). If the poor performance on the inside condition in experiment 1 was caused by a genuine impairment of conditional rule use, then the $\mathrm{PFv}+\mathrm{o}$ animals would be impaired in experiment $2 \mathrm{a}$. If the impairment on the inside condition in experiment 1 was caused by a generalization of a failure set from the other conditions, then there would be no impairment in experiment $2 \mathrm{a}$.

Experiment 2b: The effect of PFv + o lesions on transfer to a greater separation between stimuli and response boxes. Against the second conclusion drawn from experiment 1 (that $\mathrm{PFv}+\mathrm{o}$ lesions impaired performance to an even greater degree when the stimulus and action positions were separated), it might be argued that the results were made less clear by the preoperative performance of the control group being slightly, albeit nonsignificantly, worse than the preoperative performance of the $\mathrm{PFv}+\mathrm{o}$ group. Although $\mathrm{PFv}+\mathrm{o}$ performance on problems in which the stimuli and response boxes were spatially separated was at chance, whereas the decline in performance of the $\mathrm{PFv}+\mathrm{o}$ group was statistically greater than any change in control performance, and although the decline in $\mathrm{PFv}+$ o performance was statistically related to the stimuli response box separation, there was not a statistically significant three-way interaction between group, test period, and separation. Experiments 2b, 3, and 4 therefore tested whether it was possible to replicate the finding of greater impairment when stimuli and response boxes were separated in the manner of the far condition.

The data for experiment $2 \mathrm{~b}$ were collected when animals were given "transfer tests" after learning each of the four problem pairs in experiment 2a. Two transfer tests assessed the ability to perform the same problem pair but with the stimuli and response boxes spatially separated using the far condition procedure while obtaining 200 rewards (i.e., over $2 \mathrm{~d}$ ). The animals' performances on the first $2 \mathrm{~d}$ of the "far transfer test" were compared with their performances on the previous last $2 \mathrm{~d}$ of criterion responding on the same problem pair presented using the inside procedure with a saving score. Negative and positive scores indicated decrements and improvements in performance, respectively. As an additional control, after reaching criterion on the two other problem pairs in 2a (problems 2 and 4), the animals continued for an extra $2 \mathrm{~d}$. In other words, the inside procedure was used for 2 more days, and these extra sessions were referred to as "inside transfer tests." Performance of control and lesion animals on far transfer tests and inside transfer tests was then compared.

Experiment 3: the effect of PFv+o lesions on learning new conditional action-selection problems in the presence of separation between stimuli and response boxes. Experiment 2a provided clear support for the first conclusion of experiment $1: \mathrm{PFv}+\mathrm{o}$ lesions impaired visual stimulus conditional action selection even when the stimuli and response boxes were not separated. The support for the second conclusion of experiment 1 , that $\mathrm{PFv}+\mathrm{o}$ lesions impaired selection of the visual stimulus for guiding action that was provided by experiment $2 \mathrm{~b}$, was not clear cut. Although performance of $\mathrm{PFv}+\mathrm{o}$ lesion animals was statistically worse on the far transfer tests than on the inside transfer tests, the effect was small in size on the second replication. Experiment 3 therefore tested whether a relative impairment on far trials might be more robust if the problem pair 


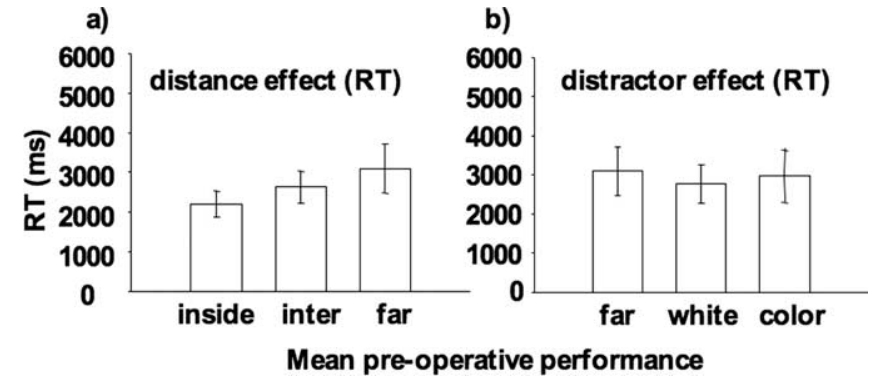

Figure 4. Experiment 1. Before surgery, RT significantly increased with increasing distance between the visual stimuli and the response boxes $(\boldsymbol{a})$, but the introduction of distractors did not lead to any additional increase in RT (b).

were novel and had not been previously encountered in the inside condition, as had been the case in experiment 2 , or if they had not previously been encountered preoperatively, as had been the case in experiment 1 . The number of errors made by control and $\mathrm{PFv}+\mathrm{o}$ lesion animals while reaching a criterion level of $85 \%$ correct while obtaining 200 rewards (i.e., over $2 \mathrm{~d}$ ) on a new far problem pair was compared with the number of errors made reaching the same level of performance on the four inside problem pairs in experiment 2 .

Experiment 4: preoperative and postoperative comparison of the effect of $P F v+o$ lesions on learning new conditional action-selection problems in the presence of separation between stimuli and response boxes. Experiment 3 suggested that spatial separation of stimulus and response boxes caused a particularly robust increase in impairment when animals were learning a novel problem pair. Experiment 4 tested whether the increased impairment was a consistent and general consequence of the $\mathrm{PFv}+\mathrm{o}$ lesion and not tied to any specific stimulus pattern. Bilateral $\mathrm{PFv}+\mathrm{o}$ lesions were made in the three animals that had previously served as controls. The effect of the lesion on new learning of novel problem pairs was tested. The ability of all three animals to learn two novel inside and two novel far problem pairs before surgery was compared with their ability to learn two novel inside and two novel far problem pairs after surgery. Learning of an additional novel inside and an additional novel far problem pair was also assessed after surgery. Testing of each problem pair was conducted in isolation, as in experiment 2 . The order in which problem pairs were presented was counterbalanced to ensure that any learning impairment under any particular set of conditions (inside or far) was not just a consequence of the order of testing.

\section{Results}

\section{Experiment 1}

Preoperative performance: the effect of separating stimuli and response boxes on action selection

After training to a criterion level of $85 \%$ correct responding, there was no obvious effect of the distance separating the stimuli from the response boxes on the level of correct responding of the five animals before any surgical intervention (mean scores for the inside, intermediate, and far conditions, $8.8,12.4$, and $13.8 \%$ ). It was nevertheless clear that greater separation between the stimuli and response boxes impinged on action selection because response times (RTs), although generally long, were modulated by the distance between the stimuli and the response boxes (Fig. 4a). RTs increased as the separation between the stimuli and the response boxes increased in the inside, intermediate, and far conditions $(F=67.96$; $\mathrm{df}=1,3 ; p=0.004)$. Longer $\mathrm{RT}$ s of this type are typically found when no premium is placed on speeded responding and the responses are made by making hand movements on a touch-screen monitor.

\section{Preoperative performance: the effect of distractors on} action selection

It was not clear, however, that the presence of distractors caused any additional difficulty in selecting the correct action. When

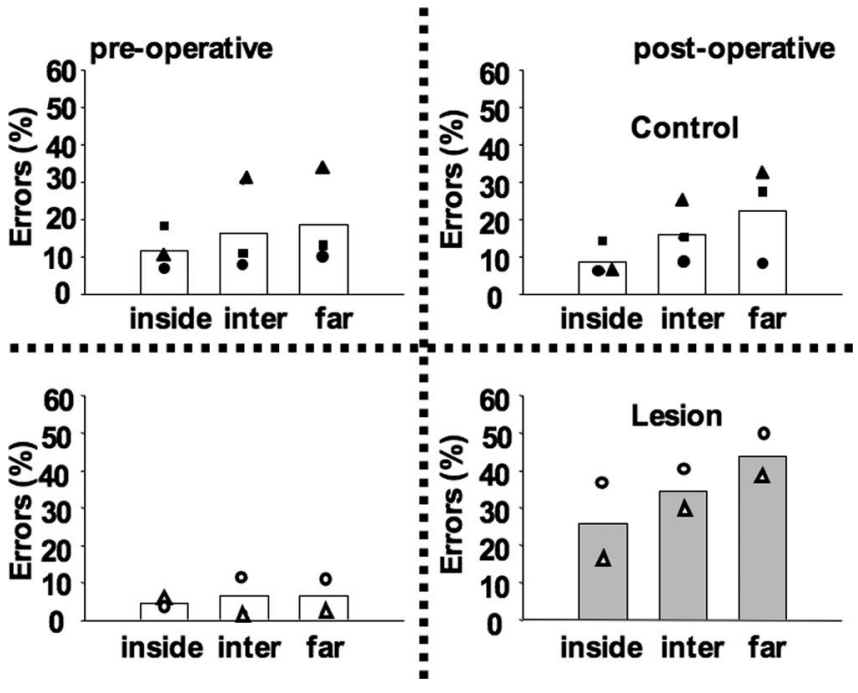

Figure 5. Experiment 1. The effect of the PFv +0 lesion on action selection and stimulus selection is illustrated by a comparison of the errors made by the lesion group (bottom) and the control group (top) before and after surgery or an equivalent time period (left and right, respectively) in the inside, intermediate, and far conditions. The gray bars are used to indicate performance by animals with lesions. The PFv + o lesion impaired performance across conditions, but the decrement in performance was significantly worse in the far condition. The open circles and triangles indicate the scores for animals PFV1 and PFV2. The filled circles, triangles, and squares indicate scores for animals PFV3, PFV4, and PFV5.

performance in the far condition was compared with performance in the two conditions, white distractor and color distractor, which entailed the same separation between stimuli and response boxes, there was no modulation $(p>0.1)$ of error rate or RT (Fig. 4b).

\section{The effect of PFv $+o$ lesions on action selection in the context of} separation of stimuli and response boxes

Error rates were increased by the $\mathrm{PFv}+\mathrm{o}$ lesion (Fig. 5). The performance of the $\mathrm{PFv}+\mathrm{o}$ lesion group declined relative to the performance of the control group after surgery (interaction of group and period, $F=67.96 ; \mathrm{df}=1,3 ; p=0.004$ ). Although there was no significant difference between the groups before surgery $(p>0.1)$, there was a significant group difference in performance after the surgery $(F=11.068 ; \mathrm{df}=1,3 ; p=0.045)$. Although there was no three-way interaction between group, period, and distance, there was a two-way interaction between period and distance $(F=5.97 ; \mathrm{df}=2,6 ; p=0.0037)$, and subsequent testing for linear contrasts confirmed that distance only interacted with testing period in the $\mathrm{PFv}+\mathrm{o}$ lesion group $(F=$ 266.27; $\mathrm{df}=1,1 ; p=0.039)$ but not in the control group $(p>$ 0.1 ). In summary, there was a general decrement in the ability to select actions that were conditional on instructing visual stimuli after the PFv+o lesion, but the impairment was significantly exacerbated by increasing the separation distance between the stimuli and contingent actions.

The effect of PFv $+o$ lesions on action selection in the context of distractors

To test for the influence of distractors on performance, a second analysis compared performance in the far condition with performance in the white distractor and color distractor conditions, which were matched for separation between stimuli and response boxes (Fig. 6). There was an interaction between group and testing period $(F=22.59$; $\mathrm{df}=1,3 ; p=0.018)$. No interaction involving the presence of any distractor approached significance $(p>0.1)$. In summary, just separating the stimuli from the re- 

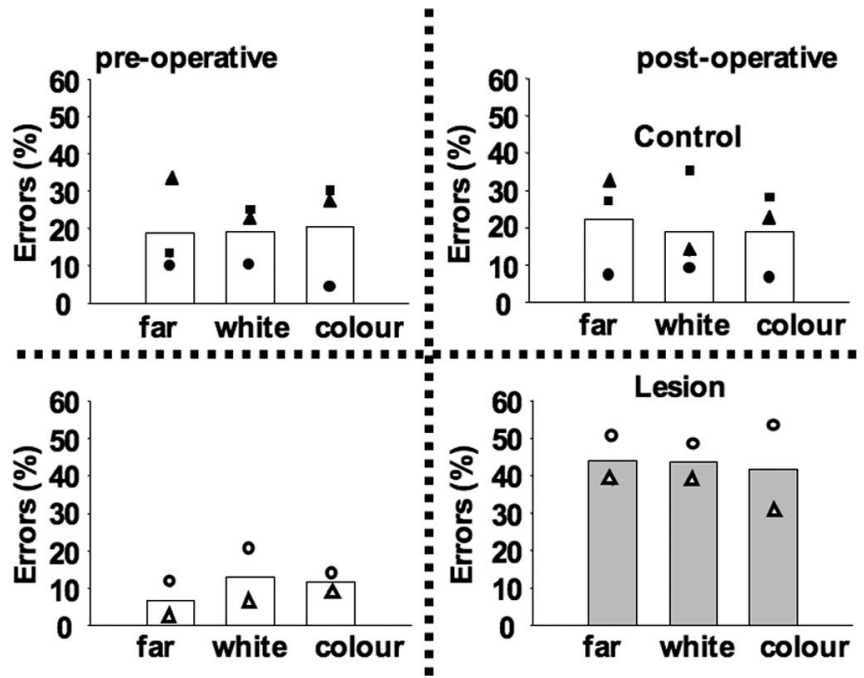

Figure 6. Experiment 1. The effect of distractors is illustrated by a comparison of the errors made by the lesion group (bottom) and the control group (top) before and after surgery or an equivalent time period (left and right, respectively) in the far, white distractor, and color distractor conditions. The gray bars are used to indicate performance by animals with lesions. The $\mathrm{PFv}+0$ lesion impaired performance uniformly across all three conditions. Animals with $\mathrm{PFv}+0$ lesions did not get significantly worse when distractors were interposed between the instructing stimuli and the response boxes; performance was already approximately at chance in the far condition even before any distractors were added. The open circles and triangles indicate the scores for animals PFV1 and PFV2. The filled circles, triangles, and squares indicate scores for animals PFV3, PFV4, and PFV5.

sponse boxes caused the animals with $\mathrm{PFv}+\mathrm{o}$ lesions considerable difficulty, but this difficulty was not worsened by the presence of distractors.

Experiment 2a: the effect of PFv+o lesions on learning new conditioned action-selection problems in the absence of any separation between stimuli and response boxes

The animals with $\mathrm{PFv}+\mathrm{o}$ lesions made more errors learning the new problem pairs. On average, the two animals with $\mathrm{PFv}+\mathrm{o}$ lesions made 145.25 and 226.75 errors, whereas $67.75,45.25$, and 13.5 errors were made by each of the three control animals on average. The difference was significant $(F=19.77 ; \mathrm{df}=1,3 ; p=$ $0.021)$. $\mathrm{PFv}+\mathrm{o}$ lesions caused a significant impairment in conditional action selection even when there was no separation between stimuli and response boxes on any trial of a testing session.

\section{Experiment 2b: the effect of PFv+o lesions on transfer to a} greater separation between stimuli and response boxes A significant one-way linear contrast between group and transfer distance $(F=8.103 ; \mathrm{df}=1,3 ; p=0.033)$ suggested that the $\mathrm{PFv}+\mathrm{o}$ lesion group was significantly more impaired than the controls when they transferred to the far distance condition than when they were given more days of the inside condition (Fig. 7). It was noticeable that the effect was small in size even if it was statistically significant; there was little decrement in performance in one of the PFv+o animals in the second far distance transfer test. It therefore seemed necessary to empirically reexamine the importance of separation between the stimuli and response boxes in an additional experiment. Because testing of new learning ability provided clear indices of impairment in experiment 3 , it seemed plausible that a more conclusive test of the importance of the separation between stimuli and response boxes might be obtained by testing the new learning of problem pairs in the inside and far conditions. This was examined in experiments 3 and 4 .

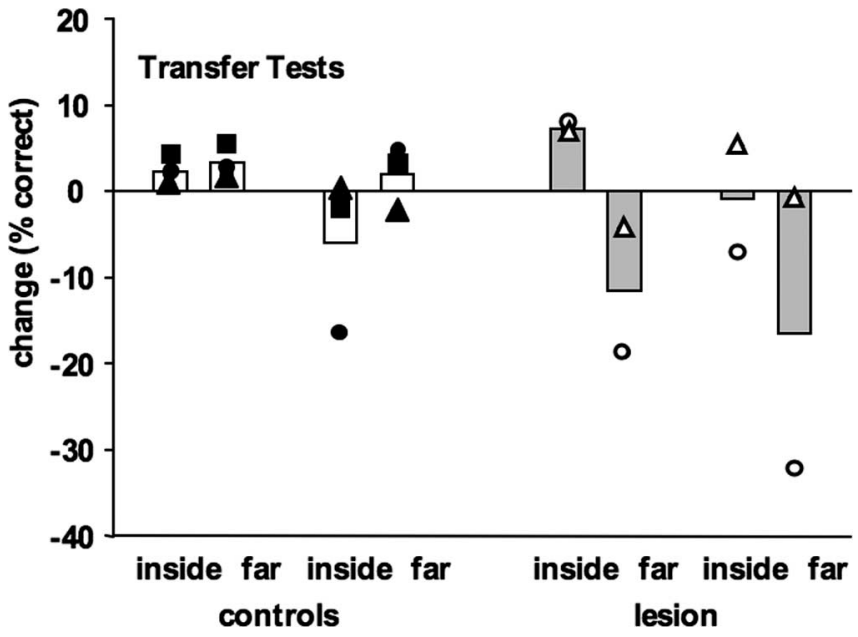

Figure 7. Experiment $2 b$. After learning four new inside problem pairs to a criterion of $85 \%$ correct, animals were tested on additional trials in the inside condition format in two cases. In two other cases, however, the animals were transferred to a version of the same problem pair in which the stimuli and response boxes were separated in the manner of the far condition. A positive score indicates a continued improvement beyond the $85 \%$ criterion, and a negative score indicates a worsening of performance below the $85 \%$ criterion. Gray bars indicate the $\mathrm{PFv}+0$ lesion group. PFv +0 animals were significantly worse when they transferred to performing the same problem pair but in the far condition format. The open circles and triangles indicate the scores for animals PFV1 and PFV2. The filled circles, triangles, and squares indicate scores for animals PFV3, PFV4, and PFV5.

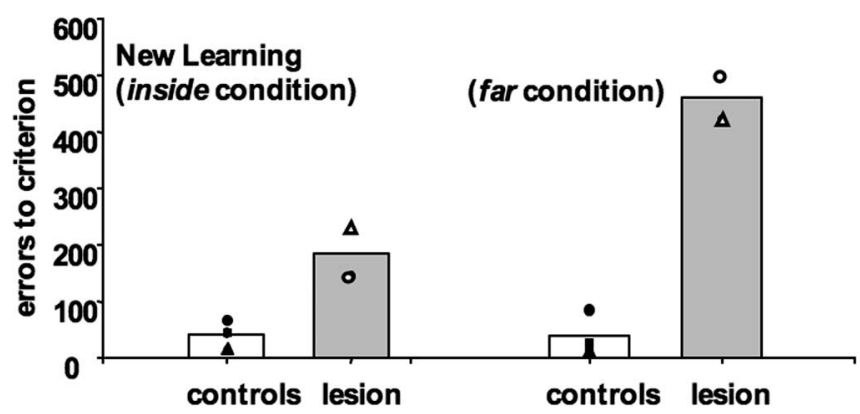

Figure 8. In experiment 2a (left), PFv +0 animals (gray bars) made significantly more errors learning new inside condition problems. The deficit confirms that PFv +0 lesions impair action selection even in the absence of interleaved trials of the more difficult intermediate, far, and distractor conditions. Data from experiment 3 are shown on the right. Animals with PFv +0 lesions made significantly more errors when learning a new far problem pair than when learning new inside problem pairs (left). The increased size of the PFv +0 deficit suggests that $\mathrm{PFv}+0$ is not just concerned with using a conditional rule to select actions, but it is also involved in the selection of the behavioral relevant stimulus. The open circles and triangles indicate the scores for animals PFV1 and PFV2. The filled circles, triangles, and squares indicate scores for animals PFV3, PFV4, and PFV5.

Experiment 3: the effect of PFv $+o$ lesions on learning new conditional action-selection problems in the presence of separation between stimuli and response boxes

The control animals did not take any longer to learn a problem pair in the far condition than they had taken on average to learn problem pairs in the inside condition, but the $\mathrm{PFv}+\mathrm{o}$ animals made more than twice as many errors (Fig. 8). The impairment of animals with $\mathrm{PFv}+\mathrm{o}$ lesions was significantly greater when learning a novel problem pair in the far condition than it was on average when learning novel problem pairs in the inside condition $(F=313.09 ; \mathrm{df}=1,3 ; p<0.001)$. 
Experiment 4: preoperative and postoperative comparison of the effect of PFv+o lesions on learning on new conditional action-selection problems in the presence of separation between stimuli and response boxes

Animals learned inside and far condition problem pairs at a similar rate before surgery (Fig. 9, left), but they were particularly slow to learn far condition problems pairs after surgery (Fig. 9, right). The only exception to this general trend was found on the first postoperative test when monkey 4 took a long time to learn an inside condition problem pair. This was the first problem that this animal encountered postoperatively. Order of testing cannot account for any other aspects of the pattern of results because near and far tests were interleaved. Because there were more postoperative data cases than preoperative data cases, the analysis was conducted by (1) looking at the interaction between testing session (the five levels corresponded to the two preoperative problem pairs and the three postoperative problem pairs) and the distance separating the stimuli and response boxes and then (2) performing subsequent separate analyses of preoperative and of postoperative tests. In the first analysis, there was a significant interaction between the five levels of session and distance $(F=$ $5.035 ; \mathrm{df}=4,8 ; p=0.025)$. An additional analysis of just the two preoperative levels of session revealed that distance exerted no significant effect on performance before $\mathrm{PFv}+\mathrm{o}$ lesion $(p>0.1)$. A third analysis showed that the effect of distance was apparent when just the three postoperative problems were considered; there was an interaction of distance and postoperative session $(F=7.535 ; \mathrm{df}=2,4 ; p=0.046)$. In summary, the three analyses demonstrate that increasing the separation between the stimuli and the response boxes impaired performance but only after the $\mathrm{PFv}+\mathrm{o}$ lesion.

\section{Discussion}

There are two distinct but not mutually exclusive accounts of $\mathrm{PFv}+\mathrm{o}$ function. Its importance for action selection that is conditional on the presence of a stimulus, instruction, or context has been emphasized in lesion (Rushworth et al., 1997; Parker and Gaffan, 1998; Bussey et al., 2001), cell recording (Asaad et al., 1998, 2000; White and Wise, 1999; Wallis et al., 2001), and neuroimaging (Toni and Passingham, 1999; Toni et al., 1999, 2001; Eliassen et al., 2003; Crone et al., 2005) studies. Conversely, cell recording (Sakagami and Niki, 1994; Rainer et al., 1998; Sakagami et al., 2001; Everling et al., 2002; Lebedev et al., 2004), lesion (Dias et al., 1996), and neuroimaging (Brass and von Cramon, 2004) studies have also suggested a PFv + o role in attentional selection because of its apparent importance for selecting the stimulus information that is relevant for the task in hand at the expense of that which is irrelevant.

It might be argued that the importance of $\mathrm{PFv}+\mathrm{o}$ for attentional selection of task-relevant information is accounted for by the rule-guided nature of paradigms used to assess selective attention. Typically, paradigms have varied which stimulus information is relevant or irrelevant during testing, and a rule determines which is relevant on a given trial. It could be claimed that animals are using a conditional rule to decide which stimulus information is relevant in a given context. In the present experiment, the difficulty of identifying the relevant stimulus was ma- nipulated in a simple way: by just spatially separating it from the response positions.

Because $\mathrm{PFv}+\mathrm{o}$ lesions impair conditional action selection more severely than discrimination learning (Parker and Gaffan, 1998; Bussey et al., 2001), it has been argued that PFv+o has a special importance for action selection rather than learning in general. Conditional tasks, however, differed from discrimination tasks not just in the conditional nature of the correct response but in terms of the difficulty of selecting task-relevant information. During discrimination, all of the information relevant to the correct choice is present at the position of the correct stimulus that the animal touches. In a conditional task, however, the correct action is conditional on information that is spatially separated from the target of the action. Although the animal's attention may be drawn to the response locations, because of their association with reward, the guiding cue information cannot be ignored if the animal is to perform well. It might, therefore, be contended that $\mathrm{PFv}+\mathrm{o}$ involvement in conditional action selection is a consequence of the importance of $\mathrm{PFv}+\mathrm{o}$ in identifying and using the task-relevant cue information.

In experiment 1 , macaques were taught visuospatial conditional action-selection problems in which they chose between two response boxes on either side of a touch-screen monitor. The stimuli for guiding action selection were present within the response boxes in the inside condition, so there was no need to identify task-relevant information at a location other than at that of the response (Fig. 1a). The $\mathrm{PFv}+\mathrm{o}$ animals were impaired although the attentional demands were no greater than in discrimination learning (Fig. 4). The impairment was not caused by a "failure set" that had generalized from the interleaved trials of the far, white distractor, and color distractor conditions, on which the lesion animals were clearly impaired (Fig. $1 b-e$ ); in experiment $2 \mathrm{a}$, the inside condition was tested in isolation, and again the $\mathrm{PFv}+\mathrm{o}$ animals were significantly impaired (Fig. 8). $\mathrm{PFv}+\mathrm{o}$ has a central role in conditional action selection even when the difficulty of identifying and using task-relevant information is kept to a minimum.

Stimulus identification and use, however, was also a determinant of impairment. Even for controls, simply separating the stimuli from the response boxes modulated RT (Fig. 4a). The conditional nature of action selection remained constant, but the difficulty of using the task-relevant stimulus was increased because attention would have been immediately drawn to response locations associated with rewards. In experiment 1, $\mathrm{PFv}+\mathrm{o}$ performance significantly declined with increasing separation of stimuli from the action location (Fig. 5). In experiment 
$2 \mathrm{~b}, \mathrm{PFv}+\mathrm{o}$ animals were significantly worse when they transferred to versions of a learned problem in which the stimuli were separated from the contingent actions (Fig. 7). Although statistically significant, the sizes of the effects were small in experiment 2b. Experiment 3, however, demonstrated a larger effect when separation between stimulus and response locations was present during initial learning (Fig. 8). The large $\mathrm{PFv}+\mathrm{o}$ impairment in learning with spatial separation could not be attributed to testing order, preoperative performance differences, or proactive interference because the effect was replicated in experiment 4 (Fig. 9).

An attempt was also made to manipulate the difficulty of selecting the task-relevant information by interposing irrelevant stimuli between relevant stimuli and the response boxes (Fig. $1 d, e)$. The attentional load imposed by irrelevant stimuli even outside a task-relevant location can make visual discrimination difficult after lesions in posterior ventral stream areas, such as temporal occipital area TEO and visual area V4 (De Weerd et al., $1999,2003)$. In the current investigations, the irrelevant distractors did not cause any additional slowing of response in the control animals beyond that already induced by separation of the stimuli and response boxes (Fig. 4b). The irrelevant distractors did not cause any additional impairment after $\mathrm{PFv}+\mathrm{o}$ lesion beyond that already seen with spatial separation of stimuli and response boxes (Fig. 6). It should be remembered, however, that $\mathrm{PFv}+\mathrm{o}$ performance was already, on average, at chance once the stimuli and response boxes were separated in the far condition.

The significant influence of stimulus response box distance is consistent with an attentional role for $\mathrm{PFv}+\mathrm{o}$. $\mathrm{PFv}+\mathrm{o}$ is a prefrontal division of a stream of areas concerned with directing the bias of attention toward relevant information (Kastner and Ungerleider, 2000) and adaptively encoding task parameters that determine successful behavior (Duncan, 2001). The effect of $\mathrm{PFv}+\mathrm{o}$ lesions is distinct to that of TEO/V4 lesions. TEO/V4 lesions make it difficult to disentangle relevant and irrelevant stimuli when they are close together and therefore within the range encompassed by a receptive field of a neuron in temporal lobe area TE. Moving the stimuli away from each other made matters better after a TEO/V4 lesion (Buffalo et al., 2005), but, in the present experiment, moving the stimuli farther from the movement targets made matters worse. At the core of the PFv $+o$ deficit is a failure to identify what information in an array is relevant for the task in hand rather than a failure to focus selectively on a relevant visual stimulus at the expense of a spatially adjacent one. The critical determinant of impairment was not difficulty of focusing on one stimulus as opposed to another, as might be the case after TEO/V4 lesion, but simply the allocation of attention to any location other than that of the response, which was closely associated with reward. The deficit may be better described as an impairment of identifying and using behaviorally relevant information rather than an impairment in selecting one visual stimulus as opposed to another as occurs after TEO/V4 damage.

It might be argued that separation of the stimulus and response locations introduced a confounding working memory element into the task. This is unlikely for two reasons. First, stimulus response box separation did not entail any delay between presentation of the stimuli and response boxes, and the stimuli were present at the time that the response was made. Second, unlike the case with dorsolateral prefrontal lesions, delay is not a critical determinant of $\mathrm{PFv}+\mathrm{o}$ lesion impairment or activation (Rushworth et al., 1997; Petrides, 2005).

Lateral frontal neuron activity, unlike more posterior or medial frontal activity, is strongly correlated with stimulus attention rather than with movement intention or expectations about the consequences of the movement (Boussaoud and Wise, 1993; Matsumoto et al., 2003; Wallis and Miller, 2003). The possibility that lateral frontal neurons might have a critical role in maintaining information in short-term memory has received considerable interest. Lebedev et al. (2004), however, have shown that activity in this region better represents the location of attended stimuli than it does the location of remembered stimuli. Within the lateral prefrontal cortex, $\mathrm{PFv}+\mathrm{o}$, rather than dorsolateral prefrontal cortex, may be more concerned with identifying behaviorally relevant signals and their implications for action (Rushworth and Owen, 1998; Hoshi and Tanji, 2004; Ninokura et al., 2004).

$\mathrm{PFv}+\mathrm{o}$ is just one part of a distributed circuit for conditional action selection, and it has been difficult to determine its special contribution. Proportionally more dorsal premotor than prefrontal neurons encode conditional rules for action selection (Wallis and Miller, 2003), and striatal neurons encode new action-selection rules more quickly than prefrontal neurons during learning (Pasupathy and Miller, 2005). Lesion studies suggest that a striatal premotor circuit may be most important for recalling old and well established conditional rules but less important for new learning (Nixon et al., 2004). In conjunction with such previous studies, the present results suggest that $\mathrm{PFv}+\mathrm{o}$ is less involved in automatization but instead that it is concerned with deployment of efficient learning strategies (Bussey et al., 2001) and in the initial registration of information that will be used to guide new conditional action contingencies.

\section{References}

Asaad WF, Rainer G, Miller EK (1998) Neural activity in the primate prefrontal cortex during associative learning. Neuron 21:1399-1407.

Asaad WF, Rainer G, Miller EK (2000) Task-specific neural activity in the primate prefrontal cortex. J Neurophysiol 84:451-459.

Boussaoud D, Wise SP (1993) Primate frontal cortex: neuronal activity following attentional versus intentional cues. Exp Brain Res 95:15-27.

Brass M, von Cramon DY (2004) Selection for cognitive control: a functional magnetic resonance imaging study on the selection of task-relevant information. J Neurosci 24:8847-8852.

Brasted PJ, Wise SP (2004) Comparison of learning-related neuronal activity in the dorsal premotor cortex and striatum. Eur J Neurosci 19:721-740.

Buffalo EA, Bertini G, Ungerleider LG, Desimone R (2005) Impaired filtering of distracter stimuli by TE neurons following V4 and TEO lesions in macaques. Cereb Cortex 15:141-151.

Bunge SA, Kahn I, Wallis JD, Miller EK, Wagner AD (2003) Neural circuits subserving the retrieval and maintenance of abstract rules. J Neurophysiol 90:3419-3428.

Bussey TJ, Wise SP, Murray EA (2001) The role of ventral and orbital prefrontal cortex in conditional visuomotor learning and strategy use in rhesus monkeys (Macaca mulatta). Behav Neurosci 115:971-982.

Bussey TJ, Wise SP, Murray EA (2002) Interaction of ventral and orbital prefrontal cortex with inferotemporal cortex in conditional visuomotor learning. Behav Neurosci 116:703-715.

Chen LL, Wise SP (1995) Neuronal activity in the supplementary eye field during acquisition of conditional oculomotor associations. J Neurophysiol 73:1101-1121.

Crone EA, Wendelken C, Donohue S, Bunge SA (2005) Dissociable brain networks for rule representation and task-switching. Cereb Cortex, in press.

De Weerd P, Peralta M, Desimone R, Ungerleider LG (1999) Loss of attentional stimulus selection after extrastriate cortical lesions in macaques. Nat Neurosci 2:753-758.

De Weerd P, Desimone R, Ungerleider LG (2003) Generalized deficits in visual selective attention after V4 and TEO lesions in macaques. Eur J Neurosci 18:1671-1691.

Dias R, Robbins TW, Roberts AC (1996) Dissociation in prefrontal cortex of affective and attentional shifts. Nature 380:69-72. 
Duncan J (2001) An adaptive coding model of neural function in prefrontal cortex. Nat Rev Neurosci 2:820-829.

Eliassen JC, Souza T, Sanes JN (2003) Experience-dependent activation patterns in human brain during visual-motor associative learning. J Neurosci 23:10540-10547.

Everling S, Tinsley CJ, Gaffan D, Duncan J (2002) Filtering of neural signals by focused attention in the monkey prefrontal cortex. Nat Neurosci 5:671-676

Hoshi E, Tanji J (2004) Area-selective neuronal activity in the dorsolateral prefrontal cortex for information retrieval and action planning. J Neurophysiol 91:2707-2722.

Kastner S, Ungerleider LG (2000) Mechanisms of visual attention in the human cortex. Annu Rev Neurosci 23:315-341.

Lebedev MA, Messinger A, Kralik JD, Wise SP (2004) Representation of attended versus remembered locations in prefrontal cortex. PLoS Biol 2:e365.

Matsumoto K, Suzuki W, Tanaka K (2003) Neuronal correlates of goalbased motor selection in the prefrontal cortex. Science 301:229-232.

Murray EA, Brasted PJ, Wise SP (2002) Arbitrary sensorimotor mapping and the life of primates. In: Neuropsychology of memory, Ed 3 (Squire LR, Schacter DL, eds), pp 339-348. New York: Guilford.

Ninokura Y, Mushiake H, Tanji J (2004) Integration of temporal order and object information in the monkey lateral prefrontal cortex. J Neurophysiol 91:555-560.

Nixon PD, McDonald KR, Gough PM, Alexander IH, Passingham RE (2004) Cortico-basal ganglia pathways are essential for the recall of wellestablished visuomotor associations. Eur J Neurosci 20:3165-3178.

Parker A, Gaffan D (1998) Memory after frontal/temporal disconnection in monkeys: conditional and non-conditional tasks, unilateral and bilateral frontal lesions. Neuropsychologia 36:259-271.

Passingham RE (1993) The frontal lobes and voluntary action. Oxford: Oxford UP.

Passingham RE, Toni I, Rushworth MFS (2000) Specialisation within the prefrontal cortex: the ventral prefrontal cortex and associative learning. Exp Brain Res 133:103-113.

Pasupathy A, Miller EK (2005) Different time courses of learning-related activity in the prefrontal cortex and striatum. Nature 433:873-876.

Paxinos G, Huang XF, Toga A (2000) The rhesus monkey brain in stereotaxic coordinates. San Diego: Academic.

Petrides M (1985) Deficits in nonspatial conditional associative learning after periarcuate lesions in the monkey. Behav Brain Res 16:95-101.
Petrides M (2005) Lateral prefrontal cortex: architectonic and functional organization. Philos Trans R Soc Lond B Biol Sci 360:781-795.

Rainer G, Asaad WF, Miller EK (1998) Selective representation of relevant information by neurons in the primate prefrontal cortex. Nature 393:577-579.

Rushworth MFS, Owen AM (1998) The functional organization of the lateral frontal cortex: conjecture or conjuncture in the electrophysiology literature? Trends Cogn Sci 2:46-53.

Rushworth MFS, Nixon PD, Eacott MJ, Passingham RE (1997) Ventral prefrontal cortex is not essential for working memory. J Neurosci 17:4829-4838.

Sakagami M, Niki H (1994) Encoding of behavioral significance of visual stimuli by primate prefrontal neurons: relation to relevant task conditions. Exp Brain Res 97:423-436.

Sakagami M, Tsutsui K-i, Lauwereyns J, Koizumi M, Kobayashi S, Hikosaka O (2001) A code for behavioural inhibition on the basis of color, but not motion, in ventrolateral prefrontal cortex of macaque monkey. J Neurosci 21:4801-4808.

Schiller PH, Chou I-H (1998) The effects of frontal eye field and dorsomedial frontal cortex lesions on visually guided eye movements. Nat Neurosci 1:248-253.

Schrier AM, Harlow HF (1957) Direct manipulation of the relevant cue on difficulty of discrimination. J Comp Physiol Psychol 50:576-592.

Toni I, Passingham RE (1999) Prefrontal-basal ganglia pathways are involved in the learning of visuomotor associations: a PET study. Exp Brain Res 127:19-32.

Toni I, Schluter ND, Josephs O, Friston K, Passingham RE (1999) Signal-, set- and movement-related activity $\mathrm{n}$ the human brain: an event-related fMRI study. Cereb Cortex 9:35-49.

Toni I, Rushworth MFS, Passingham RE (2001) Neural correlates of visuomotor associations. Spatial rules compared with arbitrary rules. Exp Brain Res 141:359-369.

Wallis JD, Miller EK (2003) From rule to response: neuronal processes in the premotor and prefrontal cortex. J Neurophysiol 90:1790-1806.

Wallis JD, Anderson KC, Miller EK (2001) Single neurons in prefrontal cortex encode abstract rules. Nature 411:953-956.

White IM, Wise SP (1999) Rule-dependent neuronal activity in the prefrontal cortex. Exp Brain Res 126:315-335.

Wise SP, Murray EA (2000) Arbitrary associations between antecedents and actions. Trends Neurosci 23:271-276. 